\title{
Effect of molar intrusion with temporary anchorage devices in patients with anterior open bite: a systematic review
}

\author{
Ahmad Saleem Alsafadi ${ }^{1 *}$, Mohannad M. Alabdullah', Humam Saltaji ${ }^{2} \mathbb{D}$, Anas Abdo ${ }^{3}$ and Mohamed Youssef ${ }^{1}$
}

\begin{abstract}
Objective: The objective of the study is to assess the effect of molar intrusion with temporary anchorage devices on the vertical facial morphology and mandibular rotation during open bite treatment in the permanent dentition.

Methods: We performed a systematic review of the published data in seven electronic databases up to September 2015. We considered studies for inclusion if they were examining the effects of posterior teeth intrusion on the vertical facial morphology with open bite malocclusion in the permanent dentition. Study selection, risk of bias assessment, and data-extraction were performed in duplicate. Meta-analysis was not possible due to dissimilarity and heterogeneity among the included studies.

Results: Out of the 42 articles that met the initial eligibility criteria, 12 studies were finally selected. Low level of scientific evidence was identified after risk of bias assessment of the included studies with no relevant randomized controlled trial performed. Out of the 12 selected studies, five studies used miniplates and seven studies used miniscrews. Mandibular counterclockwise rotation was found to be between $2.3^{\circ}$ and $3.9^{\circ}$ in six studies (as sassed by mandibular plane angle, between MeGo or GoGn and SN or FH plane) while it was less than $2^{\circ}$ in the remaining studies.

Conclusions: Current weak evidence suggests that molar intrusion with temporary anchorage devices may cause mandibular counterclockwise autorotation. Future well-conducted and clearly reported multicenter randomized controlled trials that include a non-treatment control group are needed to make robust recommendations regarding the amount of mandibular rotation during open bite treatments.
\end{abstract}

Keywords: Systematic review, Skeletal open bite, Molar intrusion

\section{Review}

\section{Introduction}

Open bite malocclusion is considered one of the most difficult orthodontic problems to correct because it appears as a result of the interaction of numerous etiological factors (genetic, dental, skeletal, functional, soft tissue, and habit) that contribute to its development [1]. An open bite can occur unilaterally or bilaterally in the buccal segments; it is particularly seen in the anterior teeth. Generally, different features have been found

\footnotetext{
* Correspondence: alsafadisaleem10@gmail.com

'Department of Orthodontics, Faculty of Dentistry, Damascus University,

Damascus, Syria

Full list of author information is available at the end of the article
}

to be associated with the skeletal anterior open bite distinguishing it from other types of malocclusion including increased lower face height, short posterior face height, [2] increased gonial and mandibular plane angles, [3] and increased maxillary molar dentoalveolar height [4]. Several reports have found correlations between orofacial muscle activity and vertical facial morphology [5-8]. These studies showed positive relationships between anterior open bite and weak musculature.

Various therapeutic approaches have been proposed for the treatment of an anterior open bite. These approaches vary depending on the causative factors and involve myotherapy, preventive treatment, functional therapy, orthognathic surgery, and orthodontic treatment using 
anterior teeth extrusion or posterior teeth intrusion [9]. Among the non-surgical orthodontic treatment methods are the temporary anchorage devices (TADs) including miniplates [10] and miniscrew or micro-screw implants $[9,11]$. Extrusion of anterior teeth is another alternative approach for open bite management, but it must take into consideration the smile esthetic [12]. Extrusion, however, is a less stable treatment than intrusion. The intrusion of posterior teeth with temporary anchorage devices was suggested to lead to decreased lower facial height by a counterclockwise rotation of the mandible; this may resemble the orthognathic surgery outcomes for any open bite patients [10].

Molar intrusion is recommended in open bite patients who usually have increased molar height [13]. While many reports indicated that increased molar height is one of the common findings in individuals with skeletal open bite [14], others do not support those findings [15, 16]. In order to evaluate the results of molar intrusion in the treatment of open bite malocclusion, it is necessary to recognize the effect of the posterior teeth intrusion on the mandibular rotation and facial morphology.

Many reports evaluated the effect of open bite treatment during mixed dentition stage [17] and [18]. A recent systematic review examined open bite treatment modalities in children found no consistent findings regarding the most effective treatment modality in growing patients with open bite malocclusion [19]. However, no comprehensive review was conducted to examine the effects of posterior teeth intrusion on vertical facial morphology in non-growing patients. Therefore, the goal of the current report is to systematically review the effect of molar intrusion with temporary anchorage devices on the vertical facial morphology and mandibular rotation during open bite treatment in the permanent dentition stage.

\section{Material and methods}

This systematic review was reported according to the principles of the PRISMA statement for reporting systematic reviews of the health sciences [20].

\section{Search strategy}

Comprehensive electronic searches up to September 30, 2015, were conducted in the following databases: PubMed, Embase, Cochrane Database of Systematic Reviews, Cochrane Central Register of Controlled Trials, Ovid, Scopus, and Web of science. The literature searches used the following Medical Subject Headings $(\mathrm{MeSH})$ terms: "molar intrusion," "posterior teeth intrusion," and "anterior open bite" which were crossed with the following terms "mandibular autorotation" and "facial morphology." In addition, the following journals were searched individually to find out any missing articles: The Angle Orthodontists, American Journal of Orthodontics and Dentofacial Orthopedics, European Journal of Orthodontics, Korean journal of orthodontics, and Journal of Orofacial Orthopedics. No restrictions were applied regarding date of publication, language, or status during database searches. The search strategy for PubMed can be found in Table 1 .

\section{Focused question}

What is the effect of molar intrusion with temporary anchorage devices on the vertical facial morphology and mandibular rotation in the permanent dentition stage during open bite treatment?

\section{Selection criteria (PICO question: population, intervention, comparison, outcome)}

The following eligibility criteria were used to determine eligible reports for this systematic review:

Population: Adolescent and adult patients with anterior open bite malocclusion. Studies examining patients with craniofacial anomalies or syndromes, cleft lip and/ or palate, surgically assisted treatment, and patients in the mixed dentition stage were excluded from the review. Only human studies were included without consideration of gender.

Intervention: Patients undergoing orthodontic treatment for open bite correction by posterior teeth intrusion in the upper and/or lower arch by using temporary anchorage devices.

Comparison: A temporary anchorage devices technique for posterior segment intrusion vs. control or another equivalent intervention, or before and after treatment. Outcomes: Angular and linear measurements used to assess the vertical changes of the mandible: overbite and maxillary and mandibular plane angle (MMA); mandibular plane angle (MPA) between Go-Gn or MeGo and reference plane (FH or SN); lower anterior facial height (LAFH), the distance between anterior nasal spine ANS and point Me; Jarabak ratio, the ratio between posterior facial height (PFH) and anterior facial height (AFH); $Y$-axis angle, the angle between SellNasion (SN) and Sell-Gnathion (S-Gn); the distance between lower first molar (L1) and mandibular plane; and the distance between upper first molar U6 and the reference plane, either palatal plane or horizontal plane. Study design: Randomized and non-randomized controlled clinical trials, clinical trials (prospective

Table 1 Search strategy in PubMed

\#1 molar intrusion OR posterior teeth intrusion OR anterior open bite

\#2 facial morphology OR mandibular autorotation

\#3 \#1 AND \#2 
and retrospective), and case series studies. Excluded articles included case reports with $\leq 8$ subjects, animal studies, review articles, abstracts, and discussions.

\section{Study selection}

The titles and abstracts of all articles obtained through the electronic searches were screened independently by three reviewers (ASA, MA, and AA). Since it cannot rely on abstracts to get enough information about the results of posterior teeth intrusion on the facial structure, no attempt at this stage was made to identify studies that did not mention the effect of molar intrusion on facial morphology and mandibular rotation. After obtaining a sufficient number of abstracts, full articles were retrieved for the final selection process. The reference list of the articles that have been retrieved was checked out for additional studies that may have been missed in the initial searches. A consensus was reached among the assessors about the articles that met the eligibility criteria.

\section{Data collection and analysis}

Data was extracted in duplicate by two reviewers (ASA and MA) on the following items: year of publication, study design, materials, method measurements, age, sample size, treatment period, force applied, amount of reduction of open bite, mandibular rotation obtained, improvement of facial morphology gained, side effects, and author's conclusions, among others.

\section{Risk of bias in individual studies}

The risk of bias of included trials was assessed using the methodological index for non-randomized trials (MINORS) tool (Table 2) [21]. Two reviewers (ASA and MA) performed the evaluations, and in cases of disagreement, consensus was reached after discussion. Methodological quality was done for each article without blinding to the authors.

\section{Data synthesis}

We planned to perform a meta-analysis if both quality and quantity of the information retrieved from the finally selected studies justified a meaningful statistical combination.

\section{Results}

Among 503 articles retrieved as a result of the initial searching process, 393 articles were excluded according to the information provided in their titles and abstracts, while 68 articles were excluded as they were duplicate articles. Consequently, 42 studies remained for the eligibility process, and eventually, only 12 studies fulfilled the inclusion criteria [10, 22-32]. One article by Hart et al. [32] was identified after the date of our search. Table 3 shows the study design and characteristics of the final selected articles. After searching manually within references of the approved articles, it was found that all related studies were included in the initial electronic search process. Figure 1 shows the scheme of article selection and the number of articles accepted and excluded. The complete list of excluded articles and reasons for exclusion are available upon request.

Different measurements have been used to determine the outcomes of the skeletal changes resulting from posterior teeth intrusion during open bite treatment. Table 4 shows linear and angular measurements mentioned in the selected reports, which indicate the amount of mandibular autorotation and the consequent improvement in facial esthetics.

All studies included in our methodological scoring process have low-moderate quality as presenting in Table 2 . Randomization, sample size evaluation, and blinding were not mentioned in any studies. Three clinical trials with control groups were found in this review [10, 22, 23]. The criteria used to assess the amount of molar intrusion and mandibular rotation with its effect on facial morphology were stated properly by nine articles $[10,22,23,25-27$, 29-32], while follow-up length stability was examined in five reports [22, 23, 28, 29, 31].

Out of the 12 selected studies, five studies [10, 22, 25, $27,28]$ used miniplates and seven studies [23, 24, 26, 29-32] used miniscrews. The amount of mandibular rotation was more than $2^{\circ}$ in six studies $[10,23-26$, 28 . The maximum amount of mandibular counterclockwise rotation was found to be $3.9^{\circ}$ [24], while the lowest amount was $1.1^{\circ}$ as reported by Hart et al. [32] by using miniscrews in the upper arch only. In the Kuroda et al. [10] study, $3.3^{\circ}$ mandibular counterclockwise rotation was achieved using temporary anchorage devices compared with the orthognathic surgery. Detailed report of outcome measurements and characteristics for each study are presented in Tables 3 and 4.

Both dissimilarity and heterogeneity was found in the outcome measures, after analyzing data in the related studies. As a result, meta-analysis was not possible for this systematic review.

\section{Discussion}

Molar intrusion is one of the valid approaches used for open bite treatment. While true molar intrusion was quantified clinically in a previous review [33], no systematic review was conducted to examine the effect of molar intrusion on the facial morphology and mandibular autorotation in the permanent dentition.

Despite progress in orthodontic treatment techniques, open bite treatment still represents a challenge for orthodontists. While orthognathic surgery is considered the gold standard for skeletal open bite treatment to achieve the optimal esthetic and occlusal result [34], non-surgical 
Table 2 Risk of bias assessment of included studies

\begin{tabular}{|c|c|c|c|c|c|c|c|c|c|c|c|c|}
\hline Quality item & $\begin{array}{l}\text { Sugawara et al. } \\
2002[22]\end{array}$ & $\begin{array}{l}\text { Deguchi et al. } \\
2011 \text { [23] }\end{array}$ & $\begin{array}{l}\text { Buschang et al. } \\
2011 \text { [24] }\end{array}$ & $\begin{array}{l}\text { Akan et al. } \\
2013 \text { [25] }\end{array}$ & $\begin{array}{l}\text { Xun et al. } \\
2007 \text { [26] }\end{array}$ & $\begin{array}{l}\text { Erverdi et al. } \\
2004 \text { [27] }\end{array}$ & $\begin{array}{l}\text { Erverdi et al. } \\
2007 \text { [28] }\end{array}$ & $\begin{array}{l}\text { Scheffler et al. } \\
2014 \text { [29] }\end{array}$ & $\begin{array}{l}\text { Foot et al. } \\
2014[30]\end{array}$ & $\begin{array}{l}\text { kuroda et al. } \\
2007[10]\end{array}$ & $\begin{array}{l}\text { Hart et al. } \\
2015 \text { [32] }\end{array}$ & $\begin{array}{l}\text { Lee and Park } \\
\text { 2008. [31] }\end{array}$ \\
\hline 1. A clear stated aim & 1 & 2 & 1 & 2 & 2 & 2 & 2 & 1 & 2 & 2 & 2 & 2 \\
\hline $\begin{array}{l}\text { 2. Inclusion of consecutive } \\
\text { patients }\end{array}$ & 0 & 2 & 2 & 0 & 0 & 0 & 0 & 2 & 1 & 0 & 2 & 0 \\
\hline $\begin{array}{l}\text { 3. Prospective collection of } \\
\text { data }\end{array}$ & 0 & 1 & 2 & 1 & 1 & 0 & 1 & 0 & 2 & 1 & 1 & 1 \\
\hline $\begin{array}{l}\text { 4. Endpoints appropriate to } \\
\text { the aim of the study }\end{array}$ & 2 & 2 & 1 & 2 & 2 & 2 & 1 & 2 & 2 & 2 & 2 & 1 \\
\hline $\begin{array}{l}\text { 5. Unbiased assessment of } \\
\text { the study end point }\end{array}$ & 0 & 0 & 0 & 0 & 0 & 0 & 0 & 0 & 0 & 0 & 0 & 0 \\
\hline $\begin{array}{l}\text { 6. Follow-up period } \\
\text { appropriate }\end{array}$ & 2 & 2 & 0 & 0 & 0 & 0 & 1 & 2 & 0 & 0 & 0 & 2 \\
\hline $\begin{array}{l}\text { 7. Loss to follow-up less than } \\
5 \%\end{array}$ & 2 & 2 & 0 & 0 & 0 & 0 & 0 & 0 & 0 & 0 & 0 & 2 \\
\hline $\begin{array}{l}\text { 8. Prospective calculation of } \\
\text { the study size }\end{array}$ & 0 & 0 & 0 & 0 & 0 & 0 & 0 & 0 & 0 & 0 & 0 & 0 \\
\hline \multicolumn{13}{|l|}{$\begin{array}{l}\text { Additional criteria in the case } \\
\text { of comparative study }\end{array}$} \\
\hline $\begin{array}{l}\text { 9. An adequate control } \\
\text { group }\end{array}$ & 2 & 2 & - & - & - & - & - & - & - & 2 & - & - \\
\hline 10. Contemporary groups & 0 & 2 & - & - & - & - & - & - & - & 2 & - & - \\
\hline $\begin{array}{l}\text { 11. Baseline equivalence of } \\
\text { groups }\end{array}$ & 0 & 1 & - & - & - & - & - & - & - & 1 & - & - \\
\hline $\begin{array}{l}\text { 12. Adequate statistical } \\
\text { analyses }\end{array}$ & 2 & 2 & 0 & 2 & 2 & 1 & 2 & 2 & 2 & 2 & 2 & 2 \\
\hline Total & 11 & 18 & 6 & 7 & 7 & 5 & 7 & 9 & 9 & 12 & 9 & 10 \\
\hline
\end{tabular}


Table 3 Characteristics of studies included in systematic review

\begin{tabular}{|c|c|c|c|c|c|c|c|}
\hline Author and year of publication & Sample size and age & Comparison & Study design & Method measurement & Study materials & Treatment time & Force applied \\
\hline Sugawara et al. (2002) [22] & SAS (9) (13.3 to 28.9 yrs) & $\begin{array}{l}\text { Pre- and post-, one group } \\
\text { only (miniplates) }\end{array}$ & $\mathrm{R}, \mathrm{CS}$ & $\begin{array}{l}\text { Lateral cephalometric } \\
\text { analysis, panoramic } \\
\text { analysis, dental cast } \\
\text { analysis }\end{array}$ & $\begin{array}{l}\text { SAS miniplate (L shaped) } \\
\text { in lower molars only }\end{array}$ & $\begin{array}{l}\text { SAS } 14.9 \text { mo (9 to } \\
22 \text { mo) follow-up } \\
12 \text { mo }\end{array}$ & Not declared \\
\hline Deguchi et al. (2011) [23] & $\begin{array}{l}\text { G1: non-IA (15) } 22.9 \text { yrs;G2: } \\
\text { IA (15) } 25.7 \text { yrs }\end{array}$ & $\begin{array}{l}\text { Two-group comparison } \\
\text { (non-implant anterior } \\
\text { elastics and HPHG with } \\
\text { MEAW vs. implant group) }\end{array}$ & $R, L, C T$ & $\begin{array}{l}\text { Lateral cephalometric } \\
\text { analysis, cast analysis } \\
\text { PAR and DI scores }\end{array}$ & $\begin{array}{l}\text { G1: non-implant group } \\
\text { (HPHG, MEAW, or } \\
\text { accentuated COS with } \\
\text { elastics);G2: implant } \\
\text { group (sectional wire } \\
\text { in upper and lower } \\
\text { posterior segment) }\end{array}$ & $\begin{array}{l}\text { G1: non- IA 1- } \\
3 \text { yrs;G2: IA 1- } \\
3 \text { yrs2 yrs follow- } \\
\text { up }\end{array}$ & Not declared \\
\hline Buschang et al. (2011) [24] & MSIs (9) 13.2 yrs & $\begin{array}{l}\text { Pre- and post-, one group } \\
\text { only (miniscrews) }\end{array}$ & $P, C S$ & $\begin{array}{l}\text { Lateral cephalometric } \\
\text { analysis }\end{array}$ & $\begin{array}{l}\text { MSIs miniscrew } \\
\text { implants in upper } \\
\text { posterior segment } \\
\text { (with RPE) + buccal } \\
\text { miniscrews in lower } \\
\text { molars }\end{array}$ & $\begin{array}{l}1.9 \mathrm{yrs}(1.4 \text { to } \\
2.5 \mathrm{yrs})\end{array}$ & $\begin{array}{l}150 \mathrm{~g} \text { per } \\
\text { side }\end{array}$ \\
\hline Akan et al. (2013) [25] & Miniplate (19) 17.7 yrs & $\begin{array}{l}\text { Pre- and post-, one group } \\
\text { only (miniplate + acrylic } \\
\text { plate) }\end{array}$ & $P, C S$ & $\begin{array}{l}\text { Lateral cephalometric } \\
\text { analysis PA radiograph } \\
\text { EMG and EVG } \\
\text { recording }\end{array}$ & $\begin{array}{l}\text { Surgical miniplates in } \\
\text { upper posterior } \\
\text { segment only }\end{array}$ & $6.8 \mathrm{mo}$ & $\begin{array}{l}400 \mathrm{~g} \text { per } \\
\text { side }\end{array}$ \\
\hline Xun et al. 2007 [26] & Miniscrews (12) 18.7 yrs & $\begin{array}{l}\text { Pre- and post-, one group } \\
\text { only (miniscrews) }\end{array}$ & $\mathrm{R}, \mathrm{CS}$ & $\begin{array}{l}\text { Lateral cephalometric } \\
\text { analysis }\end{array}$ & $\begin{array}{l}\text { Midpalatal miniscrew } \\
\text { in upper arch + buccal } \\
\text { miniscrews in lower } \\
\text { molars }\end{array}$ & $6.8 \mathrm{mo}$ & $\begin{array}{l}150 \mathrm{~g} \text { per } \\
\text { side }\end{array}$ \\
\hline Erverdi et al. (2004) [27] & Miniplate (10) 17-23 yrs & $\begin{array}{l}\text { Pre- and post-, one group } \\
\text { only (miniplate) }\end{array}$ & $\mathrm{P}, \mathrm{CS}$ & $\begin{array}{l}\text { Lateral cephalometric } \\
\text { analysis PA radiograph } \\
\text { analysis }\end{array}$ & $\begin{array}{l}\text { Miniplate (I shaped) } \\
\text { sectional wire in } \\
\text { upper posterior } \\
\text { segment only, (ext of } \\
\text { upper 1st premolar in } \\
6 \mathrm{P} \text { ) }\end{array}$ & $5.1 \mathrm{mo}$ & Not declared \\
\hline Erverdi et al. (2007) [28] & Miniplate (11) 19.5 yrs & & $P, L, C S$ & $\begin{array}{l}\text { Lateral cephalometric } \\
\text { analysis }\end{array}$ & & $\begin{array}{l}9.6 \text { mo } 1 \text { yrs } \\
\text { follow-up }\end{array}$ & $\begin{array}{l}400 \mathrm{~g} \text { per } \\
\text { side }\end{array}$ \\
\hline
\end{tabular}




\section{Scheffler et al. (2014) [29]}

Foot et al. (2014) [14]

Kuroda et al. (2007) [10]

Hart et al. (2015) [32]

Palatal miniscrews (31)

(11.6 to 55.5 yrs) 20.7 yrs

Pre- and post- one group

only (palatal miniscrews)

(21 adolescent vs. 10

adult patients)

Lee HA, and Park YC. (2008) [31]

Pre- and post- one group

$31.1 \mathrm{yrs}) 23.3 \mathrm{yrs}$

only (buccal miniscrews)

Miniplate (I shaped) In

upper posterior

segment only

Miniscrews (16 P) or miniplates (14 P) in

upper posterio

segment only

3.6-9.6 mo for

intrusion 6-

$50 \mathrm{~g}$ per

analysis

33 mo total tx

side

Sydney intrusion

Conebeam + lateral

cephalometric analysis

upper

posterior segment

Lateral cephalometric

G1: TADs sectional

wire in upper and

lower posterior

segmentG2: LeFort

osteotomy and

time

$4.91 \mathrm{mo}(2.5 \mathrm{to} \quad 500 \mathrm{~g}$ per

$7.7 \mathrm{mo})$

side

G1: $27.6 \mathrm{mo}(19-\quad$ G1: 150

$\begin{array}{ll}36 \mathrm{mo}) \mathrm{G} 2: & \text { gmG2: not } \\ 33.5 \mathrm{mo}(20- & \text { declared }\end{array}$

intraoral vertical ramus

split ramus osteotomy

$\mathrm{R}, \mathrm{CS}$

Lateral cephalometric

Bilateral perimolar

palatal miniscrews (25

p) and midpalatal

miniimplants $(6 p)$ in

upper arch only (uses

TPA + QH to control

intermolar width)

Miniscrews with

palatal rigid splint to

prevent molar tipping

sectional wire in

upper posterior

segment only.
1.3 yrs Not declared

$5.4 \mathrm{mo}$

Not declared 
Table 3 Characteristics of studies included in systematic review (Continued)

\begin{tabular}{|c|c|c|c|c|c|c|}
\hline Author and year of publication & Reduction of open bite & $\begin{array}{l}\text { Effect on mandibular } \\
\text { autorotation }\end{array}$ & $\begin{array}{l}\text { Effect on facial } \\
\text { morphology }\end{array}$ & Outcomes assessed & Side effects & Author's conclusion \\
\hline Sugawara et al. (2002) [22] & $\begin{array}{l}\uparrow \text { overbite by } 4.9 \mathrm{~mm} \downarrow \\
\text { overbite } 0.9 \mathrm{~mm} \text { after } \\
1 \text { yrs follow-up }\end{array}$ & $\begin{array}{l}\text { Counterclockwise rotation } \downarrow \text { FH/ } \\
\text { MP by } 1.3^{\circ} \uparrow \mathrm{FH} / \mathrm{MP} \text { by } 0.4^{\circ} \text { after } \\
1 \text { yrs follow-up }\end{array}$ & $\begin{array}{l}\downarrow A L F H, \downarrow \text { interlabial } \\
\text { gap and improve AP } \\
\text { jaw relation. Stable } \\
\text { profile after } 1 \text { yrs } \\
\text { follow-up. }\end{array}$ & $\begin{array}{l}\text { Overbite MP/FH } \\
\text { LAFH U6-PP L6-MP }\end{array}$ & $\begin{array}{l}27.2 \% \text { rate of relapse in } \\
\text { the } 1 \text { st molars and } 30.3 \% \\
\text { in the } 2 \text { nd molars. }\end{array}$ & $\begin{array}{l}\text { SAS is effective for open bite } \\
\text { treatment; overcorrection is necessary }\end{array}$ \\
\hline Deguchi et al. (2011) [23] & $\begin{array}{l}\mathrm{G} 1: \uparrow \mathrm{OB} \text { by } 6.5 \mathrm{~mm} \downarrow \\
0.5 \mathrm{~mm} \text { after } 2 \text { yrsG2: } \uparrow \\
\text { OB by } 6.2 \mathrm{~mm} \downarrow \\
0.8 \mathrm{~mm} \text { after } 2 \text { yrs }\end{array}$ & $\begin{array}{l}\text { G1: } \uparrow \mathrm{MP} / \mathrm{SN} \text { by } 2.7^{\circ} \text { clockwise } \\
\text { rotation, } \uparrow 0.3 \mathrm{~mm} \text { after } 2 \text { yrsG } 2 \text { : } \\
\downarrow \text { MP/SN by } 3.6^{\circ} \\
\text { counterclockwise rotation, } \uparrow 1.6^{\circ} \\
\text { after } 2 \text { yrs }\end{array}$ & $\begin{array}{l}\text { G1: } \uparrow \text { AFH and } \downarrow \text { facial } \\
\text { convexity, and } \downarrow \text { lips } \\
\text { protrusionG2: } \downarrow \text { AFH, } \\
\text { more } \downarrow \text { in facial } \\
\text { convexity, and } \downarrow \text { lips } \\
\text { protrusion } \\
\text { Disappearance of } \\
\text { incompetent lips. }\end{array}$ & $\begin{array}{l}\text { Overbite MeGo/SN } \\
\text { LAFH U6-PP L6-MP }\end{array}$ & $\begin{array}{l}\text { Less molar torque control, } \\
\text { extrusion of lower molars. } \\
1 \text { patient in } \mathrm{G} 1 \text { and } 2 \\
\text { patients in } \mathrm{G} 2 \text { showed } \\
\text { relapse after } 2 \text { yrs. }\end{array}$ & $\begin{array}{l}\text { G2 showed more relapse than G1, } \\
\text { overcorrection and myofunctional } \\
\text { therapy is recommended. Keep } \\
\text { screws longer time or use retainer } \\
\text { with occlusal stops in mandible. }\end{array}$ \\
\hline Buschang et al. (2011) [24] & Not declared & $\begin{array}{l}\downarrow \text { MPA by } 3.9^{\circ} \text { counterclockwise } \\
\text { rotation }\end{array}$ & $\begin{array}{l}\text { Chin moved forward } \\
\text { by } 2.4 \mathrm{~mm} \uparrow \mathrm{SNB} \text {, and } \\
\downarrow \text { facial convexity }\end{array}$ & MPA & $\begin{array}{l}\text { Lower molar eruption. No } \\
\text { stability information. }\end{array}$ & $\begin{array}{l}\text { Using MSIs for intrusion is effective, } \\
\text { not painful or uncomfortable. }\end{array}$ \\
\hline Akan et al. (2013) [25] & $\uparrow$ Overbite by $4.79 \mathrm{~mm}$ & $\begin{array}{l}\downarrow \mathrm{Go} G \mathrm{Gn} / \mathrm{SN} \text { by } 3.79^{\circ} \\
\text { counterclockwise rotation }\end{array}$ & $\begin{array}{l}\uparrow S N B, \downarrow L A F H, \downarrow \text { AFH, } \\
\downarrow \text { facial convexity, and } \\
\uparrow \text { upper lip/ E plane }\end{array}$ & $\begin{array}{l}\text { Overbite MP/FH } \\
\text { GoGn/SN LAFH } \\
\text { U6-HL L6-MP }\end{array}$ & $\begin{array}{l}\text { Insignificance tipping } \\
\text { molars buccally. No } \\
\text { stability information. }\end{array}$ & $\begin{array}{l}\text { Miniplate is successful Tx and has no } \\
\text { effect on TMJ and masticatory } \\
\text { muscles activity. }\end{array}$ \\
\hline Xun et al. 2007 [26] & $\uparrow$ overbite by $4.2 \mathrm{~mm}$ & $\begin{array}{l}\downarrow \mathrm{Me} \mathrm{Go} / \mathrm{SN} \text { by } 2.3^{\circ} \\
\text { counterclockwise rotation }\end{array}$ & $\begin{array}{l}\downarrow \text { AFH, } \downarrow \text { LAFH, and } \downarrow \\
\text { Ns-Sn-Pos improve- } \\
\text { ment of convex } \\
\text { profile. }\end{array}$ & $\begin{array}{l}\text { Overbite MMA } \\
\text { MeGo/SN LAFH } \\
\text { U6-PP L6-MP }\end{array}$ & No stability information. & $\begin{array}{l}\text { Miniscrew provide stable, simple, and } \\
\text { less invasive anchorage. }\end{array}$ \\
\hline Erverdi et al. (2004) [27] & $\uparrow$ overbite by $3.7 \mathrm{~mm}$ & $\begin{array}{l}\downarrow \text { Go Gn / SN by } 1.7^{\circ} \\
\text { counterclockwise rotation }\end{array}$ & $\begin{array}{l}\downarrow \text { AFH, } \uparrow \text { glabella-SN- } \\
\text { pogonion. Improve } \\
\text { smile and profile. }\end{array}$ & $\begin{array}{l}\text { Overbite MMA } \\
\text { GoGn/SN U6-PP } \\
\text { L6-MP }\end{array}$ & $\begin{array}{l}\text { Upper molars tipped } \\
\text { buccally slightly, tissue } \\
\text { inflammation and } \\
\text { irritation of cheeks. No } \\
\text { stability information. }\end{array}$ & $\begin{array}{l}\text { Minimal invasive and simple } \\
\text { technique for intrusion, long-term } \\
\text { follow-up required. }\end{array}$ \\
\hline Erverdi et al. (2007) [28] & $\uparrow$ overbite by $5.1 \mathrm{~mm}$ & $\begin{array}{l}\downarrow \mathrm{Go} G \mathrm{Gn} / \mathrm{SN} \text { by } 3.0^{\circ} \\
\text { counterclockwise rotation }\end{array}$ & $\downarrow L A F H, \uparrow S N B$ & $\begin{array}{l}\text { Overbite GoGn/SN } \\
\text { LAFH U6-PP }\end{array}$ & $\begin{array}{l}\text { Minor edema, pain, and } \\
\text { extrusion of lower molars. } \\
\text { No relapse regarding } \\
\text { rotation at } 1 \text { yrs follow-up, } \\
\text { few cases showed extru- } \\
\text { sion } 1 \text { mm of upper } \\
\text { molars. }\end{array}$ & $\begin{array}{l}\text { Therapy is effective. Longer follow-up } \\
\text { required with large number of } \\
\text { patients. }\end{array}$ \\
\hline
\end{tabular}


Scheffler et al. (2014) [29]

$\uparrow$ Overbite by $2.2 \mathrm{~mm}$

Go $\mathrm{Gn} / \mathrm{SN}$ by $1.2^{\circ}$

at debondno change at

and 2 yrs follow-up

Ly

by a fraction of a

millimeter at 1 and

2 yrs follow-up

verbite GoG

L6-GoGn

$\downarrow$ LAFH, $\downarrow$ G'SnPo'

$\downarrow \mathrm{MP} / \mathrm{SN}$ by $1.2^{\circ}$

counterclockwise rotation

counterclockwise rotation
G1: counterclock-wise rotation $\downarrow$ FH/MP by $3.3^{\circ} \mathrm{G} 2$ : counterclockwise rotation $\downarrow \mathrm{FH} / \mathrm{MP}$ by $0.3^{\circ}$

G1: $\uparrow$ overbite $6.8 \mathrm{mmG} 2: \uparrow$ overb

$7 \mathrm{~mm}$

$\uparrow$ Overbite by $3.8 \mathrm{~mm}$

$\downarrow$ FH/MP by $1.1^{\circ}$

G1: $\downarrow$ TAFH \& LAFH better morphologic improvement than surgeryG2: \TAFH, change in LAFH

Hart et al. (2015) [32] counterclockwise rotation $\uparrow 0.2$ decrease in skel $\downarrow \mathrm{LAFH}, \downarrow \mathrm{AFH} \downarrow \mathrm{PFH}$
Overbite MP/FH MMA GoGn/SN LAFH U6-PP L6-M

Overbite MP/FH LAFH U6-PP L6-MP group. No stability

\section{Overbite MP/FH \\ LAFH U6-PP U6-}

BaH L6-MP in adaptation and
Overbite MeGo/SN MP/FH AFH U6-PP

$\uparrow$ Overbite by $5.47 \mathrm{~mm}$
$0.99 \mathrm{~mm}$ after 17.4

Counterclockwise rotation $\downarrow$ Me Go/SN by $2.0^{\circ} \uparrow \mathrm{Me} \mathrm{Go} / \mathrm{SN} 0.18^{\circ}$ mo retention period $\downarrow$ AFH, pogonion

moved $2.17 \mathrm{~mm}$

esthetic with muscle

$0.38 \mathrm{~mm}$ after $17.4 \mathrm{mo}$
Extrusion of lower molars, elongation of upper an lower incisors. 15 and $22 \%$ of the patients showed relapse $(>1 \mathrm{~mm})$ in overbite at 1 and 2 yrs follow-up, respectively.

Tissue irritation, difficulty

maintaining of appliance No stability information.

Elongation of incisors in

Extrusion of lower first and second molars, dista tipping of maxillary 1st molars. No stability information.

Control vertical position of

mandibular molars. Some intrusion of maxillary canine is needed.

Effective appliance for posterior teeth intrusion with minimal reactivation, and well tolerated by patients.

Molar intrusion by skeletal anchorage is simpler and cause more rotation of mandible than surgery.

Miniscrews provide adequate anchorage for molar intrusio Adolescent patients showed more favorable mandibus showed more than adults. mandibular positions could be controllar positions could be controlled by
occlusal coverage with retainer, or full fixed appliance.

Intrusion of maxillary posterior teeth

$10.36 \%$ relapse rate for molar intrusion, and $18.10 \%$ relapse rate for overbite after 17.4-mo by using miniscrews in adult patients is an effective method for open bite correction with good stability.

Abbreviation: $P$ prospective, $R$ retrospective, $C T$ clinical trial, $C S$ case series, $L$ longitudinal, $\uparrow$ increase, $\downarrow$ decrease, ext extraction, SAS skeletal anchorage device, COS curve of Spee, tx treatment, $R P E$ rapid palatal expansion, $P A$ posterior-anterior, mo months, yrs years, $P$ patients, Me Menton, Go Gonion, Gn gnathion, SN Sella-Nasion, MP mandibular plane, FH Frakfurt horizontal, MPA mandibular plane angle, MMA maxillary expansion, $P A$ posterior-anterior, mo months, yrs years, $P$ patients, Me Menton, Go Gonion, Gn gnathion, $S N$ Sella-Nasion, $M P$ mandibular plane, $F H$ Frakfurt horit
mandibular angle, U6 upper first molar, $P P$ palatal plane, $H L$ horizontal line, BaH basion horizontal plane parallel to $F H, T P A$ transpalatal arch, $Q H$ quad helix 


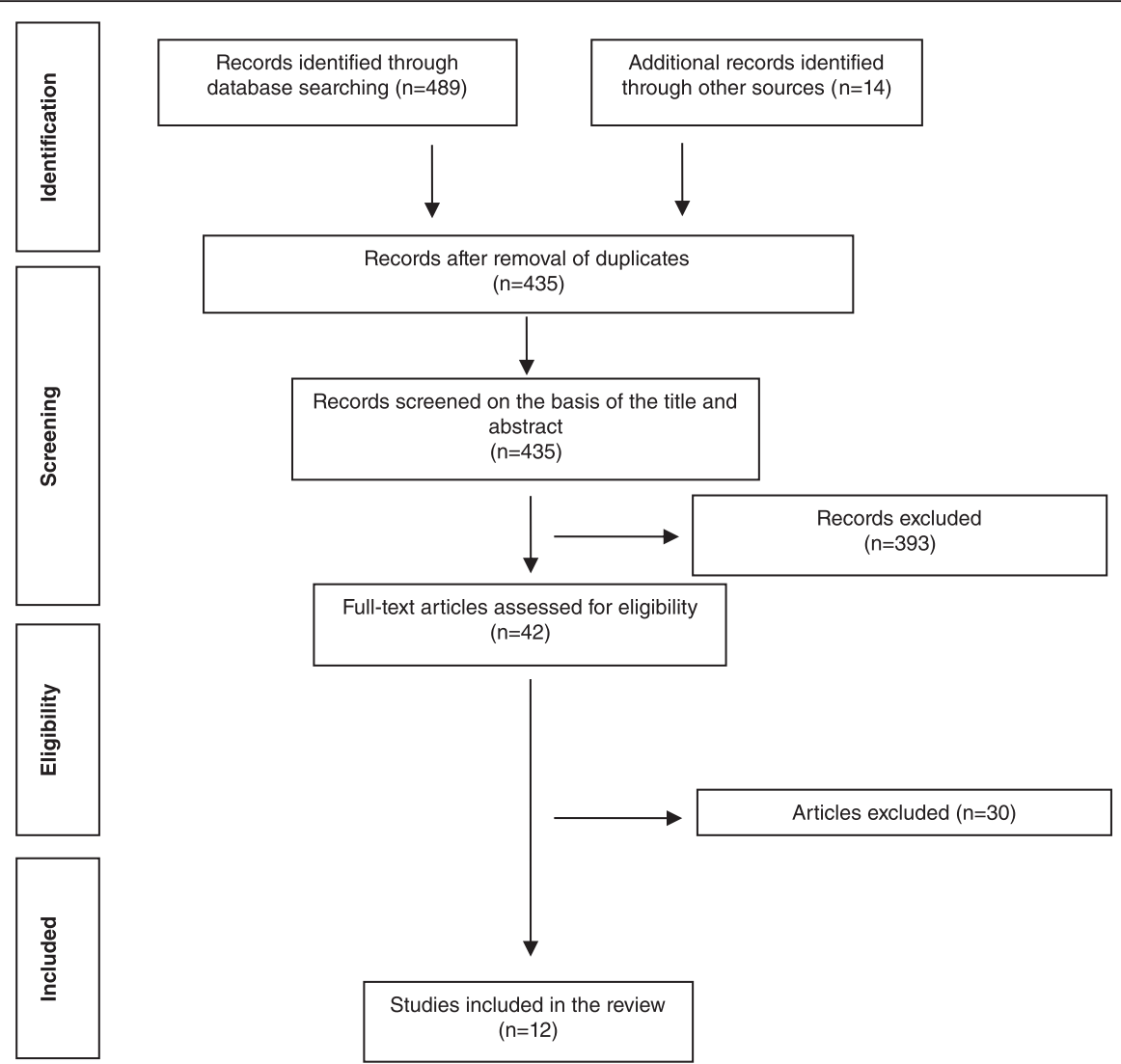

Fig 1 Flow diagram of the literature search

orthodontic treatment for these cases has become more common since it is less expensive and more acceptable for patients. Early treatment of open bite during mixed dentition has been supported by many authors [35], as it represents the importance of modification of habits or reducing the need for orthognathic surgery after puberty. Therefore, many reports were discussing open bite treatment in the younger age group. Furthermore, difficulty in obtaining open bite patients has made most reports in our electronic search are case reports.

Our systematic review identified five studies that used miniplates for molar intrusion, three of which $[25,27,28]$ applied them in the upper jaw, one study in the lower jaw [22], and one study applied TADs in both arches [10]. The applied force was $400 \mathrm{~g}$ on each side through the intrusion for a period ranged between 5.1 months [27] and 14.9 months [22]. Two reports [22, 27] did not mention the amount of applied force. Mandibular counterclockwise rotation was a common result in the miniplates groups.

Regarding posterior teeth intrusion in one arch, Akan et al. [25] and Erverdi et al. [28] showed $3.7^{\circ}$ and $3.00^{\circ}$ of mandibular counterclockwise rotation, respectively, which is higher than the value of counterclockwise rotation mentioned in the remaining two studies [22, 27], probably because of adding a posterior bite block so as to help for posterior teeth intrusion through stimulating muscular response. The amount of inter-labial gap and facial convexity was decreased after mandibular counterclockwise rotation [22, 25].

Through using miniscrews for intrusion of the lower mandibular molars and upper posterior teeth, the autorotation has increased to become $3.9^{\circ}$, which is the maximum among approved articles [24]. According to Foot et al. [30], only $1.2^{\circ}$ of mandibular rotation was achieved; this might be explained by using a subject group with an average amount of pretreatment open bites of $2.6 \mathrm{~mm}$ that required lesser amount of intrusion. Even when using a posterior occlusal splint in one group, Scheffler et al. [29] reported similar amount of mandibular rotation $\left(1.2^{\circ}\right)$. This is probably because canines remain in contact after removal of the splint; this causes a reduced degree of mandibular autorotation and correction of overbite. Hart et al. [32] reported the lowest amount of mandibular rotation $\left(1.1^{\circ}\right)$; this might be interpreted by continuous eruption of lower first and second molars. Thus, the amount of mandibular rotation would be limited as the mandibular molars eruption compensating the amount of mandibular counterclockwise rotation. These changes are more significant in adolescent patients. Moreover, facial convexity and lip protrusion decreased 
Table 4 Summary of outcome measurements of the selected studies

\begin{tabular}{|c|c|c|c|c|c|}
\hline Article & Outcomes & Before TX mean (SD) & After TX mean (SD) & Difference mean (SD) & Follow-up changes \\
\hline \multirow[t]{5}{*}{ Sugawara et al. (2002) [22] } & Overbite & $-2.8(1.8)$ & $2.1(0.8)$ & 4.9 (NA) & $1.2(0.8)$ \\
\hline & $\mathrm{MP} / \mathrm{FH}$ & $33.1(2.1)$ & $31.7(2.4)$ & $-1.3(\mathrm{NA})$ & $32.2(3.0)$ \\
\hline & L6-MP & $35.7(4.1)$ & $33.9(4.1)$ & $-1.8(\mathrm{NA})$ & $34.2(4.4)$ \\
\hline & U6-PP & $24.0(3.0)$ & $25.0(2.8)$ & $1.0(\mathrm{NA})$ & $25.1(2.5)$ \\
\hline & LAFH & $76.1(5.8)$ & $74.6(6.0)$ & $-1.5(\mathrm{NA})$ & $75.2(5.8)$ \\
\hline \multirow[t]{5}{*}{ Deguchi et al. (2011) [23] } & Overbite & $-4.4(1.2)$ & $1.8(1.1)$ & $6.2(1.7)$ & $1.0(0.9)$ \\
\hline & MP/SN & $45.8(6.0)$ & $42.2(6.7)$ & $-3.6(2.1)$ & $43.8(6.5)$ \\
\hline & U6-PP & $26.9(3.0)$ & $24.6(2.5)$ & $-2.3(1.3)$ & $25.1(2.8)$ \\
\hline & L6-MP & $36.0(2.5)$ & $35.2(1.9)$ & $-0.8(1.3)$ & $37.0(1.9)$ \\
\hline & LAFH & $74.7(5.9)$ & $72.2(5.1)$ & $-2.6(2.5)$ & $72.2(5.1)$ \\
\hline Buschang et al. (2011) [24] & MPA & NR & NR & $-3.9(1.8)$ & NA \\
\hline \multirow[t]{6}{*}{ Akan et al. (2013) [25] } & Overbite & $-3.2(1.3)$ & $1.5(1.3)$ & $4.7(1.3)$ & NA \\
\hline & GoGn/SN & $45.5(7.3)$ & $41.7(7.2)$ & $-3.7(1.8)$ & \\
\hline & $\mathrm{MP} / \mathrm{FH}$ & $36.3(7.0)$ & $33.0(6.9)$ & $-3.2(1.5)$ & \\
\hline & LAFH & $57.0(4.9)$ & $52.8(4.6)$ & $-4.1(1.7)$ & \\
\hline & U6-HL & $74.6(4.4)$ & $71.3(4.2)$ & $-3.3(1.2)$ & \\
\hline & L6-MP & $32.0(3.2)$ & $31.2(3.3)$ & $-0.8(0.8)$ & \\
\hline \multirow[t]{6}{*}{ Xun et al. (2007) [26] } & Overbite & $-2.2(0.9)$ & $2.0(0.3)$ & $4.2(0.9)$ & NA \\
\hline & MMA & $36.8(4.8)$ & $34.3(4.2)$ & $-2.5(0.9)$ & \\
\hline & MP/SN & $45.6(5.8)$ & $43.3(5.1)$ & $-2.3(0.8)$ & \\
\hline & LAFH & $82.2(3.8)$ & $80.6(3.4)$ & $-1.6(0.9)$ & \\
\hline & U6-PP & $26.3(2.2)$ & $24.5(2.0)$ & $-1.8(0.7)$ & \\
\hline & L6-MP & $37.4(3.4)$ & $36.2(3.3)$ & $-1.2(0.8)$ & \\
\hline \multirow[t]{5}{*}{ Erverdi et al. (2004) [27] } & Overbite & $-0.6(2.2)$ & $3.1(0.9)$ & $3.7(2.4)$ & NA \\
\hline & MMA & $36.9(5.4)$ & $34.7(4.5)$ & $-2.2(1.2)$ & \\
\hline & GoGn/SN & $46.5(6.0)$ & $44.8(6.7)$ & $-1.7(2.0)$ & \\
\hline & U6-PP & $26.4(1.6)$ & $23.8(2.7)$ & $-2.6(1.3)$ & \\
\hline & L6-MP & $32.8(2.7)$ & $31.7(3.1)$ & $-0.1(0.7)$ & \\
\hline \multirow[t]{4}{*}{ Erverdi et al. (2007) [28] } & Overbite & $-4.0(\mathrm{NA})$ & $1.2(\mathrm{NA})$ & $5.1(2.0)$ & NA \\
\hline & GoGn/SN & $42.5(\mathrm{NA})$ & 39.5 (NA) & $-3.0(1.5)$ & \\
\hline & LAFH & $76.5(\mathrm{NA})$ & $73.6(\mathrm{NA})$ & $-2.9(1.3)$ & \\
\hline & U6-PP & $22.6(\mathrm{NA})$ & $19.0(\mathrm{NA})$ & $-3.6(1.4)$ & \\
\hline \multirow[t]{5}{*}{ Scheffler et al. (2014) [29] } & Overbite & NR & NR & $2.2(1.6)$ & $-0.4(1.1)$ \\
\hline & GoGn/SN & & & $-1.2(1.0)$ & $0.0(0.8)$ \\
\hline & LAFH & & & $-1.6(2.2)$ & $-0.3(1.4)$ \\
\hline & U6-PP & & & $-2.3(1.4)$ & $0.5(1.2)$ \\
\hline & L6-GoGn & & & $0.6(1.6)$ & $-0.3(1.3)$ \\
\hline \multirow[t]{7}{*}{ Foot et al. (2014) [30] } & Overbite & $-2.2(1.7)$ & $0.8(1.1)$ & $3.0(1.5)$ & NA \\
\hline & MMA & $31.8(3.8)$ & 30.7 (4.3) & $-1.0(1.3)$ & \\
\hline & MP/SN & $36.1(4.9)$ & $34.9(5.3)$ & $-1.2(1.3)$ & \\
\hline & $\mathrm{MP} / \mathrm{FH}$ & $27.8(3.5)$ & 26.9 (3.6) & $-0.9(1.2)$ & \\
\hline & LAFH & $61.8(4.3)$ & $60.9(4.6)$ & $-0.9(1.1)$ & \\
\hline & U6-PP & $20.6(2.3)$ & $17.7(2.4)$ & $-2.9(0.8)$ & \\
\hline & L6-MP & $27.9(2.9)$ & $28.0(2.9)$ & $0.1(0.4)$ & \\
\hline
\end{tabular}


Table 4 Summary of outcome measurements of the selected studies (Continued)

\begin{tabular}{|c|c|c|c|c|c|}
\hline \multirow[t]{5}{*}{ Kuroda et al. (2007) [10] } & Overbite & $-5.2(1.8)$ & $1.5(0.6)$ & $6.8(1.7)$ & \multirow[t]{5}{*}{ NA } \\
\hline & $\mathrm{MP} / \mathrm{FH}$ & $38.8(6.4)$ & $35.5(6.9)$ & $-3.3(1.5)$ & \\
\hline & LAFH & $78.6(5.5)$ & $75.0(4.7)$ & $-3.6(1.8)$ & \\
\hline & U6-PP & $28.7(2.8)$ & $26.4(2.4)$ & $-2.3(2.0)$ & \\
\hline & L6-MP & $38.0(1.8)$ & $36.7(1.6)$ & $-1.3(1.2)$ & \\
\hline \multirow[t]{6}{*}{ Hart et al. (2015) [32] } & Overbite & $-3.0(1.9)$ & $0.8(1.4)$ & $3.8(0.9)$ & \multirow[t]{6}{*}{ NA } \\
\hline & $\mathrm{MP} / \mathrm{FH}$ & $32.4(6.3)$ & $31.3(6.9)$ & $-1.1(0.09)$ & \\
\hline & LAFH & $73.3(7.4)$ & $71.8(7.1)$ & $-1.5(0.03)$ & \\
\hline & U6-PP & $23.7(3.3)$ & $21.4(3.2)$ & $-2.3(0.06)$ & \\
\hline & U6-BaH & $27.4(3.7)$ & $25.7(3.7)$ & $-1.7(0.10)$ & \\
\hline & L6-MP & $30.4(3.1)$ & $31.5(3.3)$ & $1.1(0.05)$ & \\
\hline \multirow[t]{5}{*}{ Lee and Park. (2008) [31] } & Overbite & $-3.7(1.7)$ & $1.7(0.7)$ & $5.4(1.8)$ & $0.7(0.7)$ \\
\hline & $\mathrm{MeGo} / \mathrm{SN}$ & $44.9(3.9)$ & $42.9(4.5)$ & $-2.0(1.7)$ & $43.0(4.8)$ \\
\hline & $\mathrm{MP} / \mathrm{FH}$ & $37.9(3.1)$ & $35.0(3.6)$ & $-2.8(2.0)$ & $35.9(3.8)$ \\
\hline & $\mathrm{AFH}$ & $133.4(5.4)$ & $130.8(5.7)$ & $-2.6(1.9)$ & $131.1(5.4)$ \\
\hline & U6-PP & $26.7(1.2)$ & $24.5(1.7)$ & $-2.2(1.7)$ & $24.7(1.6)$ \\
\hline
\end{tabular}

significantly after posterior teeth intrusion by miniscrews $[23,24,26,31]$. Miniscrews were placed in the upper and lower arch for molar intrusion; the applied force ranged between $150 \mathrm{~g}$ on each side $[24,26,29]$ and $500 \mathrm{~g}$ per side [30]. Deguchi et al. [23] suggested that conventional treatment without miniscrews could not result in improvement in facial profile as if the miniscrews were applied for intrusion. The multiloop edgewise archwire (MEAW) technique had been the most common treatment before invention of TADs. Deguchi et al. [23] reported that using the MEAW technique improved overbite without achieving mandibular autorotation or intrusion in the upper or lower molars. The increase in overbite was because of anterior intermaxillary elastics.

The magnitude of the mandibular autorotation after molar intrusion was dependent on a set of interrelated factors including amount of intrusive force, duration of intrusion, and place of intrusion in upper or lower arch. With respect to the amount of intrusive force, it has been reported that using $400 \mathrm{~g}$ per segment for posterior tooth intrusion using miniplates will lead to $2^{\circ}$ to $4^{\circ}$ of mandibular counterclockwise rotation measured between mandibular plane $(\mathrm{GoGn})$ and SN plane $[25,28]$. Similar to the result of the upper and lower molar intrusion simultaneously by measuring the angle between mandibular plane (MeGo) and SN or FH, after application of a $150 \mathrm{~g}$ to each side by means of miniscrews $[24,26]$ or with combination with miniplates [10], the longest period for molar intrusion was achieved by applying miniplates in lower arch only for intrusion [22]. However, the intrusion time was ranged between 5 and 10 months [25-29, 31]. Increasing both the amount of intrusion force and miniscrews number will lead to decrease in the duration of intrusion to 4.9 months [30]. Generally, intrusion of the upper and lower molars simultaneously will increase the amount of the mandibular rotation and correction of the open bite $[10,23]$. It seems that using an acrylic plate along with the miniplate for upper molar intrusion will lead to intrusion of the lower molars [25, 27]; this was not confirmed in other studies [29] and [30]. Although the amount of intrusion in the lower arch was statistically non-significant, it could assist in the mandibular counterclockwise rotation. Intrusion of upper posterior teeth by means of temporary anchorage devices without acrylic plate will lead to overeruption of lower molars [32]. Occlusal coverage with a vacuum retainer or full fixed appliances with lower molar engagement could mitigate the effect of uncontrolled eruption of mandibular molars.

Comparing pre- and post-treatment measurements in the consecutive radiographs might not be a valid method to evaluate the linear and angular changes of the facial structures. Radiograph superimposition is considered an accurate method for facial changes assessment as a result of growth or orthodontic treatment and that after registration on the stable reference areas [36]. Three reports [10, 24, 27] used cephalometric superimposition without details of the reference structures. Anterior contour of the chin, inner contour of the cortical plates at the inferior border of the symphasis, and contours of the mandibular canal are well documented as stable structures for serial superimposition [37]. Mandibular autorotation has been assessed by the angular position of the mandibular plane (MP) relative to the 
FH plane $[10,22,31,32]$ or to the cranial base $(\mathrm{SN})$ [23, 25-30]. However, none of the studies measured the Jarabak ratio and $Y$-axis angle. Others used MMA to evaluate maxillary or mandibular rotation [26, 27, 30].

Improvement of facial esthetics by decreasing LAFH was mentioned in most studies except those conducted by Buschang et al. [24] and Erverdi et al. [27]. Soft tissue changes must be quantified carefully in order to assess proper changes in them. The use of $3 \mathrm{D}$ is a promising method to evaluate soft tissue changes during open bite treatment provided that they are compared with the control group and obtain an accurate measurement.

\section{Limitations}

There are no randomized clinical trials performed focused on the open bite treatment using temporary anchorage devices. Presence of randomization is an important issue to consider when determining the best treatment modality for posterior teeth intrusion. It is clinically important to investigate the amount of mandibular rotation during open bite treatment by means of miniscrews and/or miniplates in comparison with other therapeutic treatment options (such as MEAW, premolars extraction, high-pull headgear, and orthognathic surgery), as well as evaluation of the long-term stability of posterior teeth intrusion by different techniques. The drawbacks in most of the articles such as absence of untreated control groups, short follow-up period, small sample size, and presence of confounding factors should be avoided in future studies so as to reach a more accurate conclusion concerning open bite treatment.

\section{Conclusions}

Current available evidence suggests that that posterior teeth intrusion in the permanent dentition stage using TADs might cause mandibular counterclockwise rotation and improve facial esthetics. Miniscrews showed $2.3^{\circ}$ to $3.9^{\circ}$ of mandibular counterclockwise rotation (as sassed by mandibular plane angle, between MeGo or GoGn and SNFH plane) when an intrusive force applied to both upper and lower molars, which is almost similar to what was observed after application of the high intrusive force in the upper posterior segment only by means of miniplate and acrylic bite block. Absence of a standardized method of intrusion, outcome measurements, and differences in the protocols followed for molar intrusion (in one arch or both arches) have led to concluding weak clinical evidence. Future well-conducted and clearly reported multicenter randomized controlled trials with a non-treatment control group are needed to provide the best scientific evidence relating to the effect of molar intrusion on the mandibular rotation and facial esthetic during open bite treatment.

\section{Competing interests}

The authors declare that they have no competing interests.

\section{Authors' contributions}

ASA, MA, and AA collected and analyzed the data; ASA, MA, and AA assessed the risk of bias of included studies; ASA, MA, and AA drafted the manuscript and integrated the critical feedback from the other authors. All of the authors were involved in interpretation of the data. All of the authors provided feedback on the revisions to the manuscript. All authors read and approved the final manuscript.

\section{Author details}

${ }^{1}$ Department of Orthodontics, Faculty of Dentistry, Damascus University, Damascus, Syria. ${ }^{2}$ Orthodontic Graduate Clinic, Faculty of Medicine and Dentistry, University of Alberta, Edmonton, Canada. ${ }^{3}$ Faculty of Dentistry, Damascus University, Damascus, Syria.

Received: 10 January 2016 Accepted: 23 February 2016

Published online: 23 March 2016

\section{References}

1. Baek MS, Choi YJ, Yu HS, Lee KJ, Kwak J, Park YC. Long-term stability of anterior open-bite treatment by intrusion of maxillary posterior teeth. Am J Orthod Dentofacial Orthop. 2010;138(4):396 e1-9.

2. Lopez-Gavito G, Wallen TR, Little RM, Joondeph DR. Anterior open-bite malocclusion: a longitudinal 10-year postretention evaluation of orthodontically treated patients. Am J Orthod Dentofacial Orthop. 1985;87(3):175-86.

3. Kim $\mathrm{YH}$. Anterior openbite and its treatment with multiloop edgewise archwire. Angle Orthod. 1987;57(4):290-321.

4. Schudy FF. Vertical growth versus anteroposterior growth as related to function and treatment. Angle Orthod. 1964;34(2):75-93.

5. Lowe AA. Correlations between orofacial muscle activity and craniofacial morphology in a sample of control and anterior open-bite subjects. Am J Orthod Dentofacial Orthop. 1980;78(1):89-98.

6. Alabdullah M, Saltaji H, Abou-Hamed H, Youssef M. Association between facial growth pattern and facial muscle activity: a prospective cross-sectional study. Int Orthod. 2015;13(2):181-94.

7. Alabdullah MM, Saltaji H, Abou-Hamed H, Youssef M. The relationship between molar bite force and incisor inclination: a prospective crosssectional study. Int Orthod. 2014;12(4):494-504.

8. Saltaji H, Flores-Mir C, Major PW, Youssef M. The relationship between vertical facial morphology and overjet in untreated class II subjects. Angle Orthod. 2012;82(3):432-40. doi:10.2319/050711-322.1.

9. Park HS, Kwon OW, Sung JH. Nonextraction treatment of an open bite with microscrew implant anchorage. Am J Orthod Dentofacial Orthop. 2006; 130(3):391-402.

10. Kuroda S, Sakai Y, Tamamura N, Deguchi T, Takano-Yamamoto T. Treatment of severe anterior open bite with skeletal anchorage in adults: comparison with orthognathic surgery outcomes. Am J Orthod Dentofacial Orthop. 2007:132(5):599-605.

11. Hwang DH, Park KH, Kwon YD, Kim SJ. Treatment of class II open bite complicated by an ankylosed maxillary central incisor. Angle Orthod. 2011; 81(4):726-35

12. Pikdoken L, Erkan M, Usumez S. Gingival response to mandibular incisor extrusion. Am J Orthod Dentofacial Orthop. 2009;135(4):432 e1-6.

13. Kucera J, Marek I, Tycova H, Baccetti T. Molar height and dentoalveolar compensation in adult subjects with skeletal open bite. Angle Orthod. 2011; 81(4):564-9

14. Uribe F, Nanda R. Management of open bite malocclusion. In: Nanda R, editor. Biomechanics and esthetic strategies in clinical orthodontics. St Louis: Elsevier; 2005. p. 156-76.

15. Bjork A. Prediction of mandibular growth rotation. Am J Orthod Dentofacial Orthop. 1969:55(6):585-99.

16. Nahoum HI. Vertical proportions: a guide for prognosis and treatment in anterior open-bite. Am J Orthod Dentofacial Orthop. 1977;72(2):128-46.

17. Lentini-Oliveira Débora A, Carvalho Fernando R, Rodrigues Clarissa G, Ye Q, Hu R, Minami-Sugaya H, et al. Orthodontic and orthopaedic treatment for anterior open bite in children. Cochrane Database Syst Rev. 2014. doi:10. 1002/14651858.CD005515.pub3. 
18. Cozza P, Mucedero M, Baccetti T, Franchi L. Early orthodontic treatment of skeletal open-bite malocclusion: a systematic review. Angle Orthod. 2005; 75(5):707-13.

19. Feres MF, Abreu LG, Insabralde NM, Almeida MR, Flores-Mir C. Effectiveness of the open bite treatment in growing children and adolescents. A systematic review. Eur J Orthod. 2015 Jul 1. [Epub ahead of print].

20. Liberati A, Altman DG, Tetzlaff J, Mulrow C, Gøtzsche PC, loannidis JPA, et al. The PRISMA statement for reporting systematic reviews and meta-analyses of studies that evaluate health care interventions: explanation and elaboration. J Clin Epidemiol. 2009;62(10):e1-34.

21. Slim K, Nini E, Forestier D, Kwiatkowski F, Panis Y, Chipponi J. Methodological index for non-randomized studies (minors): development and validation of a new instrument. ANZ J Surg. 2003;73(9):712-6.

22. Sugawara J, Baik UB, Umemori M, Takahashi I, Nagasaka H, Kawamura H, et al. Treatment and posttreatment dentoalveolar changes following intrusion of mandibular molars with application of a skeletal anchorage system (SAS) for open bite correction. Int J Adult Orthodon Orthognath Surg. 2002;17(4): 243-53.

23. Deguchi T, Kurosaka H, Oikawa H, Kuroda S, Takahashi I, Yamashiro T, et al. Comparison of orthodontic treatment outcomes in adults with skeletal open bite between conventional edgewise treatment and implant-anchored orthodontics. Am J Orthod Dentofacial Orthop. 2011:139(4 Suppl):S60-8.

24. Buschang PH, Carrillo R, Rossouw PE. Orthopedic correction of growing hyperdivergent, retrognathic patients with miniscrew implants. J Oral Maxillofac Surg. 2011;69(3):754-62.

25. Akan S, Kocadereli I, Aktas A, Tasar F. Effects of maxillary molar intrusion with zygomatic anchorage on the stomatognathic system in anterior open bite patients. Eur J Orthod. 2013;35(1):93-102.

26. Xun C, Zeng X, Wang X. Microscrew anchorage in skeletal anterior openbite treatment. Angle Orthod. 2007;77(1):47-56.

27. Erverdi N, Keles A, Nanda R. The use of skeletal anchorage in open bite treatment: a cephalometric evaluation. Angle Orthod. 2004;74(3):381-90.

28. Erverdi N, Usumez S, Solak A, Koldas T. Noncompliance open-bite treatment with zygomatic anchorage. Angle Orthod. 2007;77(6):986-90. doi:10.2319/101206-422.1.

29. Scheffler NR, Proffit WR, Phillips C. Outcomes and stability in patients with anterior open bite and long anterior face height treated with temporary anchorage devices and a maxillary intrusion splint. Am J Orthod Dentofacial Orthop. 2014;146(5):594-602

30. Foot R, Dalci O, Gonzales C, Tarraf NE, Darendeliler MA. The short-term skeleto-dental effects of a new spring for the intrusion of maxillary posterior teeth in open bite patients. Prog Orthod. 2014;15:56.

31. Lee HA, Park YC. Treatment and posttreatment changes following intrusion of maxillary posterior teeth with miniscrew implants for open bite correction. Korean J Orthod. 2008;38(1):31-40.

32. Hart TR, Cousley RR, Fishman LS, Tallents RH. Dentoskeletal changes following mini-implant molar intrusion in anterior open bite patients. Angle Orthod. 2015;85(6):941-8.

33. Ng J, Major PW, Flores-Mir C. True molar intrusion attained during orthodontic treatment: a systematic review. Am J Orthod Dentofacial Orthop. 2006;130(6):709-14.

34. Reichert I, Figel P, Winchester L. Orthodontic treatment of anterior open bite: a review article-is surgery always necessary? J Oral Maxillofac Surg. 2014;18(3):271-7.

35. Huang GJ, Justus R, Kennedy DB, Kokich VG. Stability of anterior openbite treated with crib therapy. Angle Orthod. 1990;60(1):17-24.

36. Bishara SE, Athanasiou AE. Cephalometric methods for assessment of dentofacial changes. In: Athanasiou AE, editor. Othodontic Cephalometry. 1st ed. St Louis: Mosby-Wolfe; 1995. p. 105-24.

37. Bjork A, Skieller V. Superimposition of profile radiographs by the structural method. In: normal and abnormal growth of the mandible. Eur J Orthod. 1983;5:40-6.

\section{Submit your manuscript to a SpringerOpen ${ }^{\circ}$ journal and benefit from:}

- Convenient online submission

- Rigorous peer review

- Immediate publication on acceptance

- Open access: articles freely available online

- High visibility within the field

- Retaining the copyright to your article

Submit your next manuscript at $\gg$ springeropen.com 